\title{
Exact Fuzzy Observer for a Baker's Yeast Fed-Batch Fermentation Process
}

\author{
Enrique Herrera, Bernardino Castillo, Member, IEEE, Jesús Ramírez and Eugénio C. Ferreira
}

\begin{abstract}
The purpose of this work is to design an exact fuzzy observer for a bioprocess switching between two different metabolic states. A fed-batch baker's yeast culture is modeled by two sub-models: a respiro-fermentative state with ethanol production and a respirative state with ethanol consumption. An exact fuzzy observer model using sector nonlinearity was built for both nonlinear models; the observer gains were designed using Linear Matrix Inequalities (LMI's). The observer dynamics shows a very good tracking behavior with respect of the states of the switching partial models. The observer premise variables depend on the state variables estimated by the fuzzy observer.
\end{abstract}

\section{INTRODUCTION}

The measurement of biological parameters as the cell, byproduct concentrations and the specific growth rate is essential to the successful monitoring and control of bioprocesses [1]. However, on-line measurements of all the state variables of a bioprocess are not always available, due to the fact that: sensors are expensive, are not completely reliable and are not always sterilizable, among other facts. A state observer may be used to reconstruct, at least partially the states variables of the process. This situation has encouraged the searching of new software sensors in bioprocesses. Fed-batch cultures are used to produce high concentrations of a desired product avoiding undesirable effects such as substrate inhibition and catabolite repression. Different application of observers and parameter estimators are reported in the literature [2], [3] and [4], among others.

In processes with uncertainties and poor known kinetics, fuzzy logic may help to compensate the lack of information by adding the human expertise about the process. Different fuzzy logic applications to bioprocesses can be found in the scientific literature. For instance, Azevedo et al. [5] proposed a state observer based on a hybrid model, where the specific kinetic reactions are approximated using fuzzy

Bernardino Castillo is with the Centro de Investigación y de Estudios Avanzados del I.P.N., Unidad Guadalajara, Av. Científica 1145, colonia el bajío, C.P.45010, Zapopan, México. (email: toledo@gdl.cinvestav.mx).

Enrique Herrera is with the Centro de Investigación y de Estudios Avanzados del I.P.N., Unidad Guadalajara. On Ph. D. studies leave from the Centro de Investigación y Asistencia en Tecnología y Diseño del Estado de Jalisco A.C. (email: eherrera@ciatej.net.mx).

Jesús Ramírez is with the Centro de Investigación y Asistencia en Tecnología y Diseño del Estado de Jalisco A.C., Av. Normalistas 800, C.P. 44270, Guadalajara, México. (email: jramirez@ciatej.net.mx).

Eugénio C. Ferreira is with the IBB-Institute for Biotechnology and Bioengineering, Centre of Biological Engineering, Universidade do Minho 4710-057 Braga, Portugal. (email: ecferreira@deb.uminho.pt). inference systems, other applications are reported in [6], [7] and $[8]$.

In the case where the nonlinear model of the process is known, a fuzzy system can be used. A first approach can be done using the Takagi-Sugeno (TS) fuzzy model [9] the consequent part of the fuzzy rules are replaced by linear systems. This can be attained, for example, linearizing the model around operational points, getting local linear representation of the nonlinear system. Another way for obtaining TS models can be achieved using the method of sector nonlinearities, which allows constructing an exact fuzzy model from the original nonlinear system by means of linear subsystems [10]. From this exact model, a state observer may be designed based on the linear subsystems.

Along this line of reasoning, in this work a fuzzy state observer based on sector nonlinearities is proposed and applied to a fed-batch baker's yeast process. An interesting feature of this model is the splitting in two different partial models: a respiro-fermentative (RF) model with ethanol production and the respirative (R) model with ethanol consumption. The switching condition depends on whether the process is consuming or producing ethanol. The observer premise variables depend on the estimated variables by the fuzzy observer. The use of fuzzy observers obtained from an exact fuzzy model, applied to fed-batch culture described by partial models has not been, to the best authors' knowledge, reported in the literature.

\section{PRELIMINARIES ON FuZzy MODELS}

\section{A. Takagi-Sugeno Fuzzy Models}

The Takagi-Sugeno fuzzy models are used to represent nonlinear dynamics by means of a set of IF-THEN rules. The consequent parts of the rules are local linear systems obtained from specific information about the original nonlinear plant. The $i$ th rule of a continuous fuzzy model has the following form:

Rule i:

$$
\begin{aligned}
& \text { IF } z_{1}(t) \text { is } M_{1}^{i} \text { and ...and } z_{p}(t) \text { is } M_{p}^{i} \\
& \text { THEN }\left\{\begin{array}{l}
x(t)=A_{i} x(t)+B_{i} u(t) \\
y(t)=C_{i} x(t)
\end{array} \quad i=1, \ldots, r .\right.
\end{aligned}
$$


where $M_{j}^{i}$ is a fuzzy set and $r$ is the number of rules in the fuzzy model; $x(t) \in R^{n}$ is the state vector, $u(t) \in R^{m}$ is the input vector, $y(t) \in R^{q}$ is the output vector, $A_{i} \in R^{n \times n}, B_{i} \in$ $R^{n \times m}, y \quad C_{i} \in R^{q \times n}$ are suitable matrices, and $z(t)=$ $\left[z_{1}(t), \ldots, z_{p}(t)\right]$ is a known vector of premise variables which may depend partially on the state $x(t)$. Given a pair of $(x(t)$, $u(t)$ and using a singleton fuzzifier, a product inference and a center average engine, the aggregate TS fuzzy model can be inferred as:

$$
\begin{gathered}
x(t)=\frac{\sum_{i=1}^{r} \varpi_{i}(z(t))\left\{A_{i} x(t)+B_{i} u(t)\right\}}{\sum_{i=1}^{r} \varpi_{i}(z(t))} \\
=\sum_{i=1}^{r} h_{i}(z(t))\left\{A_{i} x(t)+B_{i} u(t)\right\}, \\
y(t)=\frac{\sum_{i=1}^{r} \varpi(z(t)) C_{i} x(t)}{\sum_{i=1}^{r} \varpi(z(t))} \\
=\sum_{i=1}^{r} h_{i}(z(t)) C_{i} x(t),
\end{gathered}
$$

where

$$
\varpi_{i}(z(t))=\prod_{j=1}^{p} M_{j}^{i}\left(z_{j}(t)\right), \quad h_{i}(z(t))=\frac{\varpi_{i}(z(t))}{\sum_{i=1}^{r} \varpi_{i}(z(t))},
$$

for all $t$. The term $M_{j}^{i}\left(z_{j}(t)\right)$ is the membership value of $z_{j}(t)$ in $M_{j}^{i}$. Since

$$
\varpi_{i}(z(t)) \geq 0 \text { and } \sum_{i=1}^{r} \varpi_{i}(z(t))>0, \quad i=1, \ldots, r,
$$

we have that

$$
h_{i}(z(t)) \geq 0 \text { and } \sum_{i=1}^{r} h_{i}(z(t))=1, \quad i=1, \ldots r,
$$

for all $t$.

\section{B. Fuzzy Observers}

The state of a system is not always fully available, so it is necessary to use an observer to reconstruct, at least partially the states variables of the process. This requires to satisfy that

$$
\lim _{t \rightarrow 0}(x(t)-\hat{x}(t))=0
$$

where $\hat{x}(t)$ denotes the state vector estimated by the fuzzy observer. There are two cases for fuzzy observers design depending on whether or not $z(t)$ depends on the state variables estimated by a fuzzy observer [10]. Given the TS fuzzy model (1), the $i$ th rule of a continuous fuzzy observer can be constructed as:

\section{Observer Rule i}

$$
\begin{aligned}
& \text { IF } z_{1}(t) \text { is } M_{1}^{i} \text { and ... and } z_{p}(t) \text { is } M_{p}^{i} \\
& \text { THEN } \\
& \hat{x}=\sum_{i=1}^{r} h_{i}(z(t))\left\{A_{i} \hat{x}(t)+B_{i} u(t)+K_{i}(y(t)-y(t))\right\} \\
& \hat{y}(t)=h_{i}(z(t)) C_{i} \hat{x}(t) \text {. }
\end{aligned}
$$

where $K_{i}$ is the observer gain for the $i$ th subsystem. If $z(t)$ depends on the estimated state variables, the observer takes the following form:

$$
\begin{gathered}
\hat{x}=\sum_{i=1}^{r} h_{i}(\hat{z}(t))\left\{A_{i} \hat{x}(t)+B_{i} u(t)+K_{i}(y(t)-\hat{y}(t))\right\}, \\
\hat{y}(t)=h_{i}(\hat{z}(t)) C_{i} \hat{x}(t) .
\end{gathered}
$$

\section{THE BAKER’s YEAST MODEL}

A fed-batch baker's yeast culture is represented by the following model

$$
\dot{x}(t)=\left(\begin{array}{c}
\left(\mu_{s}^{o}+\mu_{s}^{r}+\mu_{e}^{o}\right) x_{1} \\
\left(-k_{1} \mu_{s}^{o}-k_{2} \mu_{s}^{r}\right) x_{1} \\
\left(k_{3} \mu_{s}^{r}-k_{4} \mu_{e}^{o}\right) x_{1} \\
\left(-k_{5} \mu_{s}^{o}-k_{6} \mu_{e}^{o}\right) x_{1}+O T R
\end{array}\right)+\left(\begin{array}{c}
-x_{1} \\
-x_{2}+S_{i n} \\
-x_{3} \\
-x_{4}
\end{array}\right) D
$$

with the additional equation

$$
\dot{x}_{5}=F
$$

The variables and parameter values used in (2) are shown in table I.

TABLE I

PARAMETERS AND VARIABLES USED IN THE BAKER'S YEAST MODEL.

\begin{tabular}{l|c}
\hline \multicolumn{1}{c}{ Variable / parameter } & units \\
\hline$x_{1}=$ Biomass & $\mathrm{g} / \mathrm{l}$ \\
$x_{2}=$ Substrate & $\mathrm{g} / \mathrm{l}$ \\
$x_{3}=$ Ethanol & $\mathrm{g} / \mathrm{m} / 1$ \\
$x_{4}=$ Dissolved oxygen & $\mathrm{mg}$ \\
$x_{5}=$ Volume & $\mathrm{L}$ \\
$F=$ Flow rate & $\mathrm{L} / \mathrm{h}$ \\
$D=F / x_{5}=$ Dilution rate & $1 / \mathrm{h}$ \\
$S_{i n}=$ Inlet substrate concentration & $\mathrm{g} / 1$ \\
$\mu_{s}^{o}, \mu_{s}^{r}$ and $\mu_{e}^{o}=$ Specific growth rates & $1 / \mathrm{h}$ \\
\hline \hline
\end{tabular}


The yield coefficients values for $k_{1}, k_{2}, k_{3}, k_{4}, k_{5}, k_{6}$ are described in [11]. The oxygen transfer rate is given by OTR $=K_{L} a\left(C^{\text {sat }}-x_{4}\right)$, where $K_{L} a$ is the mass transfer coefficient and $C^{\text {sat }}$ is the oxygen saturation concentration. OTR may be split in two terms, one that is constant and the other one depends on the dissolved oxygen.

$$
K_{L} a C^{c a t} \quad \text { (3) }-K_{L} a x_{4},
$$

Pormeleau [12] suggested a reformulation of model (2) using two partial models: a respiro-fermentative partial model $(\mathrm{RF})$ with ethanol production and a respirative partial model (R) with ethanol consumption. With this reformulation a split process model is obtained switching from the RF partial model to the $\mathrm{R}$ partial model and vice versa, depending on whether the system is consuming or producing ethanol. To precise the ideas, consider a nonlinear system described by the model (2), which can be written as

$$
\begin{aligned}
& \dot{x}(t)=f_{i}(x(t))+g(x) u(t)+d \\
& y(t)=h(x(t))
\end{aligned}
$$

where $f_{i}(x(t))$ describe both the RF and R partial models, namely

$$
f_{1}=\left[\begin{array}{c}
\left(\mu_{s_{-} R F}^{o}+\mu_{s_{-} R F}^{r}\right) x_{1} \\
\left(-k_{1} \mu_{s_{-} R F}^{o}-k_{2} \mu_{s_{-} R F}^{r}\right) x_{1} \\
k_{3} \mu_{s_{-} R F}^{r} x_{1} \\
-k_{5} \mu_{s_{-} R F}^{o} x_{1}-K_{L} a x_{4}
\end{array}\right]:=f_{R F}
$$

and for the R model

$$
f_{2}=\left[\begin{array}{c}
\left(\mu_{s_{-} R}^{o}+\mu_{e_{-} R}^{o}\right) x_{1} \\
-k_{1} \mu_{s_{-} R}^{o} x_{1} \\
-k_{4} \mu_{e_{-} R}^{o} x_{1} \\
\left(-k_{5} \mu_{s_{-} R}^{o}-k_{6} \mu_{e_{-} R}^{o}\right) x_{1}-K_{L} a x_{4}
\end{array}\right]:=f_{R}
$$

and $u(t)=D=F / x_{5}$.

The input matrix $g(x)$ for both models is given by:

$$
g(x)=\left[-x_{1},-x_{2}+S_{i n},-x_{3},-x_{4}\right]^{T}
$$

As previously stated, OTR was split in two terms the first one (3) was included in the models (5) and (6) and the second one (4) is considered as a constant perturbation, and thus

$$
d=\left[\begin{array}{llllll}
0 & 0 & 0 & K l a C^{c a t}
\end{array}\right]^{T}
$$

The specific rates for the $\mathrm{RF}$ partial model are given by:

$$
\mu_{s_{-} R F}^{\circ}=Y_{O 2}\left(q_{0}^{\max } \frac{x_{4}}{K o+x_{4}}\right)
$$

$$
\mu_{s_{-} R F}^{r}=\operatorname{Yr}\left(q_{s}^{\max } \frac{x 2}{K s+x 2}-q_{o}^{\max } \frac{x_{4}}{K o+x_{4}} \frac{Y_{O 2}}{Y_{O}}\right) .
$$

For the $\mathrm{R}$ partial model the specific rates are given by

$$
\mu_{s_{-} R}^{o}=Y_{O}\left(q_{s}^{\max } \frac{x_{2}}{K s+x_{2}}\right)
$$

and $\mu_{e_{-} R}^{o}$, which can take the following values

$$
\mu_{\mathrm{e}_{-} \mathrm{R}}^{\circ}=\left\{\begin{array}{lll}
q_{e_{1}} & I F & q_{e_{1}}<q_{e_{2}} \\
q_{e_{2}} & I F & q_{e_{1}} \geq q_{e_{2}}
\end{array}\right.
$$

where

$$
\begin{gathered}
q_{e_{1}}=Y_{e} q_{e}^{\max } \frac{x_{3}}{K e+x_{3}} \frac{K i}{K i+x_{2}} \\
q_{e_{2}}=Y_{O 2} e\left(q_{o}^{\max } \frac{x_{4}}{K o+x_{4}}-\frac{Y_{O}}{Y_{O 2}}\left(q_{s}^{\max } \frac{x_{2}}{K s+x_{2}}\right)\right)
\end{gathered}
$$

From equation (11) and (12) it can be inferred that the $\mathrm{R}$ partial model given by (6) should be split in two new models: (Rqe1) when $\mu_{e_{-} R}^{o}=q_{e_{1}}$ and (Rqe2) when $\mu_{e_{-} R}^{o}=q_{e_{2}}$. The switching condition between the RF and $\mathrm{R}$ partial model, as well as, the parameters definition and values $\left(\mathrm{Ks}, \mathrm{Ki}, \mathrm{Ke}, \mathrm{Ko}, \mathrm{Yo}, \mathrm{Yr}, \mathrm{Yo}, \mathrm{Y}_{\mathrm{O} 2}, Y_{\mathrm{O} 2 e}, q_{s}^{\max }, q_{0}^{\max }\right.$ and $q_{e}^{\max }$ ) shown on equations 8 to 12 are described in [11] and [13]. To change between the RF to the $\mathrm{R}$ models, $\mathrm{F}$ was varied according to figure 1 , while $S_{i n}$ was set to 10 .

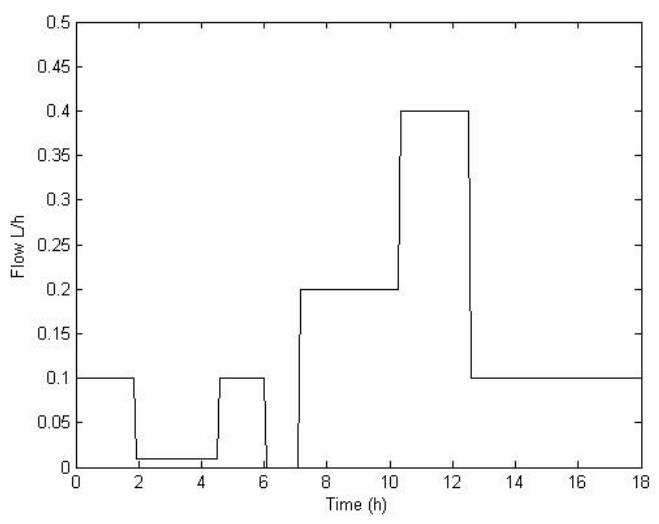

Fig. 1. The feeding flow signal

\section{The Exact Fuzzy ModeL}

When the nonlinear dynamic model for the baker's yeast is known, as well as all their parameters, a fuzzy exact model can be derived from the given nonlinear model. This requires a sector nonlinearity approach [10]. To construct the $\mathrm{RF}$ and $\mathrm{R}$ exact fuzzy models, $f_{R F}$ can be expressed as: 
RF model

$$
f_{R S}=\left[\begin{array}{cccc}
\frac{x_{4}}{K O+x_{4}} q_{o}^{\max }\left(Y_{O 2}-Y_{1} \frac{Y_{O 2}}{Y_{O}}\right) & Y_{r} q_{s}^{\max } \frac{x_{1}}{K S+x_{2}} & 0 & 0 \\
\frac{x_{4}}{K O+x_{4}} q_{o}^{\max }\left(-k_{1} Y_{O 2}+k_{2} Y_{1} \frac{Y_{O 2}}{Y_{O}}\right) & -k_{2} Y_{r} q_{s}^{\max } \frac{x_{1}}{K_{S}+x_{2}} & 0 & 0 \\
-k_{3} Y_{r} q_{o}^{\max } \frac{Y_{O 2}}{Y_{O}} \frac{x_{4}}{X_{O}+x_{4}} & k_{3} Y_{r} q_{s}^{\max } \frac{x_{1}}{K_{S}+x_{2}} & 0 & 0 \\
-k_{5} Y_{O 2} q_{o}^{\max } \frac{x_{4}}{K O+x_{4}} & 0 & 0 & -K l a
\end{array}\right]\left[\begin{array}{c}
x_{1} \\
x_{2} \\
x_{3} \\
x_{4}
\end{array}\right],
$$

and according to (11) and (12) $f_{R}$ should be split to

Rqel model

$$
f_{R_{q e l}}=\left[\begin{array}{cccc}
q_{e}^{\max } K i \frac{x_{3}}{\left(K e+x_{3}\right)\left(K i+x_{2}\right)} & Y_{o} q_{s}^{\max } \frac{x_{1}}{K S+x_{2}} & 0 & 0 \\
0 & -k_{1} Y_{o} q_{s}^{\max } \frac{x_{1}}{K S+x_{2}} & 0 & 0 \\
-k_{4} Y_{e} q_{e}^{\max } K i \frac{x_{3}}{\left(K e+x_{3}\right)\left(K i+x_{2}\right)} & 0 & 0 & 0 \\
-k_{6} Y_{e} q_{e}^{\max } K i \frac{x_{3}}{\left(K e+x_{3}\right)\left(K i+x_{2}\right)} & -k_{5} Y_{o} q_{s}^{\max } \frac{x_{1}}{K S+x_{2}} & 0 & -K l a
\end{array}\right]\left[\begin{array}{c}
x_{1} \\
x_{2} \\
x_{3} \\
x_{4}
\end{array}\right]
$$

Rqe 2 model

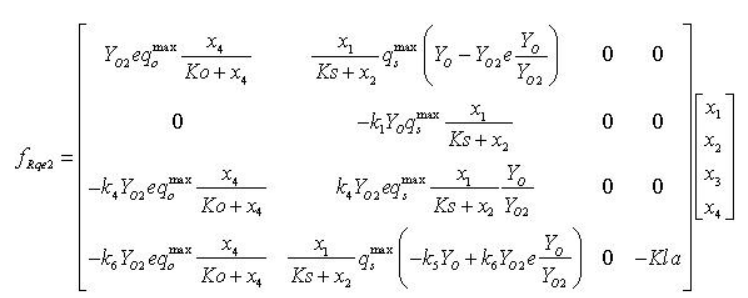

From (13-15) the fuzzy exact model using sector nonlinearities can be constructed. The premise variables are chosen as:

$$
\begin{gathered}
z_{1}(t)=\frac{x_{4}}{K o+x_{4}} \quad(16) \quad z_{2}(t)=\frac{x_{1}}{K s+x_{2}} \\
z_{3}(t)=\frac{x_{3}}{\left(K e+x_{2}\right)\left(K i+x_{3}\right)}
\end{gathered}
$$

and from (7) the premise variables for the input matrix $g(x)$ are given by:

$$
\begin{gathered}
z_{x_{1}}(t)=x_{1}, \quad z_{x_{2}}(t)=x_{2}, \\
z_{x_{3}}(t)=x_{3}, \quad z_{x_{4}}(t)=x_{4} .
\end{gathered}
$$

With these premise variables, assuming the ranges $x_{1}(t) \epsilon$ $[0,10], x_{2}(t) \in[0,1], x_{3}(t) \in[0,5]$ and $x_{4}(t) \in[0,0.007]$ and using the procedure described in [10], the membership function for $M_{l i}\left(z_{l}(t)\right)$ for the RF partial model is obtained as

$$
M_{11}\left(z_{1}(t)\right)=\frac{z_{1}(t)-a_{2}}{a_{1}-a_{2}}, M_{12}\left(z_{1}(t)\right)=\frac{-z_{1}(t)+a_{1}}{a_{1}-a_{2}},
$$

The membership functions $M_{2 j}\left(z_{2}(t)\right), M_{3 k}\left(z_{x l}(t)\right), M_{4 l}\left(z_{x 2}(t)\right)$, $M_{5 m}\left(z_{x 3}(t)\right)$ and $M_{6 n}\left(z_{x 4}(t)\right)$ are obtained following the same procedure. The maximum and minimum values for the premise variables are shown in the table II. A general fuzzy rule to infer all the fuzzy rules for the RF partial model can be stated as:

IF $z_{l}(t)$ is " $M_{l i}\left(z_{l}(t)\right)$ " and $z_{2}(t)$ is " $M_{2 j}\left(z_{2}(t)\right)$ " and $z_{x l}(t)$ is " $M_{3 k}\left(z_{x l}(t)\right)$ " and $z_{x 2}(t)$ is " $M_{4 l}\left(z_{x 2}(t)\right)$ " and $z_{x 3}(t)$ is $M_{5 m}\left(z_{x 3}(t)\right)$ and $z_{x 4}(t)$ is " $M_{6 n}\left(z_{x 4}(t)\right)$ "

\section{THEN}

$$
\begin{aligned}
& \dot{x}^{R F}(t)=A_{i j k m n}^{R F} x(t)+B_{i j k l m n}^{R F} u(t)+d \\
& i, j, k, l, m, n=1,2 .
\end{aligned}
$$

TABLE II

MAXIMUM AND MINIMUM VALUES FOR THE PREMISE VARIABLES.

\begin{tabular}{c|c|c}
\hline \hline $\begin{array}{c}\text { Premise } \\
\text { variable }\end{array}$ & $\max$ & $\mathrm{min}$ \\
\hline$z_{1}^{R F, R g e 2}(t)$ & $\mathrm{a}_{1}=0.9859$ & $\mathrm{a}_{2}=0.1$ \\
$z_{2}^{R F, \text { Rge1,Rge2 }}(t)$ & $\mathrm{b}_{1}=50$ & $\mathrm{~b}_{2}=0.1$ \\
$z_{3}^{R g e 1}$ & $\mathrm{a}_{1}=9.8039$ & $\mathrm{a}_{2}=0.1$ \\
$z_{x_{1}}(t)$ & $\mathrm{c}_{1}=10$ & $\mathrm{c}_{2}=0$ \\
$z_{x_{2}}(t)$ & $\mathrm{d}_{1}=1$ & $\mathrm{~d}_{2}=0$ \\
$z_{x_{3}}(t)$ & $\mathrm{e}_{1}=5$ & $\mathrm{e}_{2}=0$ \\
$z_{x_{4}}(t)$ & $\mathrm{f}_{1}=0.007$ & $\mathrm{f}_{2}=0$ \\
\hline \hline
\end{tabular}

The linear subsystems $A_{i j k m n}^{R F}, B_{i j k l m n}^{R F}$ are derived from

$$
A_{j k h m}^{R F}=\left[\begin{array}{cccc}
a_{i} q_{o}^{\max }\left(Y_{O 2}-Y_{r} \frac{Y_{O 2}}{Y_{O}}\right) & Y_{r} q_{s}^{\max } b_{j} & 0 & 0 \\
a_{i} q_{o}^{\max }\left(-k_{1} Y_{O 2}+k_{2} Y_{r} \frac{Y_{O 2}}{Y_{O}}\right) & -k_{2} Y_{r} q_{s}^{\max } b_{j} & 0 & 0 \\
-k_{3} a_{i} Y_{r} q_{o}^{\max } \frac{Y_{O 2}}{Y_{O}} & k_{3} Y_{r} q_{s}^{\max } b_{j} & 0 & 0 \\
-k_{5} a_{i} Y_{O 2} q_{o}^{\max } & 0 & 0 & -K l a
\end{array}\right]
$$

$i, j, k, l, m, n=1,2$.

$$
B_{i j k l m n}^{R F}=\left[-c_{k},-d_{l}+S_{i n},-e_{m},-f_{n}\right]^{T}
$$

$i, j, k, l, m, n=1,2$.

Taking into account that there are 6 premise variables in the RF partial model, there will be $2^{6}=64$ linear subsystems that are constructed from the combination of (19) and (20). The final aggregated RF model turns to be:

$$
\begin{aligned}
& \dot{x}^{R F}(t)=\sum_{1}^{64} h_{\psi}(z(t))\left\{A_{i j k m n n}^{R F} x(t)+B_{i j k l m n}^{R F} u(t)+d\right\} \\
& \left.y^{R F}(t)=\sum_{1}^{64} h_{\psi}(z(t)) C x(t)\right)
\end{aligned}
$$


where

$$
\begin{aligned}
\psi= & n+2(m-1)+4(l-1)+8(k-1) \\
& +16(j-1)+32(i-1), \\
h_{\psi}(z(t))= & M_{1 i}\left(z_{1}(t)\right) M_{2 j}\left(z_{2}(t)\right) M_{3 k}\left(z_{x_{1}}(t)\right) \\
& \times M_{4 l}\left(z_{x_{2}}(t)\right) M_{5 m}\left(z_{x_{3}}(t)\right) M_{6 n}\left(z_{x_{4}}(t)\right)
\end{aligned}
$$

This fuzzy model exactly represents the RF partial nonlinear model in the region $x_{1}(t) \in[0,10], x_{2}(t) \in[0,1]$, $x_{3}(t) \in[0,5]$ and $x_{4}(t) \in[0,0.007]$. The fuzzy exact model for the models Rqel and Rqe2 were constructed following the same procedure. It is worth to remark that also 64 subsystems are obtained for each partial model Rqel and Rqe2.

\section{THE EXACT FUZZY OBSER VER}

After an exact fuzzy model for the nonlinear baker's yeast partial model has been obtained, a fuzzy observer can now be designed. The following assumptions are made:

H1. The yield coefficients $k_{1}, k_{2}, k_{3}, k_{4}, k_{5}$ and $k_{6}$ are constant and known.

$\mathrm{H} 2$. The ethanol and the dissolved oxygen concentration are known.

When the ethanol and the dissolved oxygen are measured on line all the states variables are observable, so a full state observer can be built, the procedure described by [10] is followed. For the RF model the premise variable $z_{1}(t), z_{x 3}(t)$ and $z_{x 4}(t)$ are taken as in (16) and (20). However for $z_{2}(t)$, $z_{x 1}(t)$ and, $z_{x 2}(t)$ we have to consider the estimates, namely

$$
\hat{z}_{2}(t)=\frac{\hat{x}_{1}}{K S+\hat{x}_{2}}, \quad \hat{z}_{x_{1}}(t)=\hat{x}_{1}, \quad \hat{z}_{x_{2}}(t)=\hat{x}_{2},
$$

thus the membership functions, for $M_{2 j}\left(z_{2}(t)\right), M_{3 k}\left(z_{x l}(t)\right)$ $M_{41}\left(z_{x 2}(t)\right)$, are then modified as

$$
\begin{gathered}
M_{21}\left(\hat{z}_{2}(t)\right)=\frac{\hat{z}_{2}(t)-b_{2}}{b_{1}-b_{2}}, M_{22}\left(\hat{z}_{2}(t)\right)=\frac{-\hat{z}_{2}(t)+b_{1}}{b_{1}-b_{2}}, \\
M_{31}\left(\hat{z}_{x_{1}}(t)\right)=\frac{\hat{z}_{x_{1}}(t)-c_{2}}{c_{1}-c_{2}}, M_{32}\left(\hat{z}_{x_{1}}(t)\right)=\frac{-\hat{z}_{x_{1}}(t)+c_{1}}{c_{1}-c_{2}}, \\
M_{41}\left(\hat{z}_{x_{2}}(t)\right)=\frac{\hat{z}_{x_{2}}(t)-d_{2}}{d_{1}-d_{2}}, M_{42}\left(\hat{z}_{x_{2}}(t)\right)=\frac{-\hat{z}_{x_{2}}(t)+d_{1}}{d_{1}-d_{2}},
\end{gathered}
$$

The linear subsystems $A_{i j k \text { m } m n}^{R F}, B_{i j k l m n}^{R F}$ for the observer are also derived from (21) and (22). The fuzzy rules for the RF partial model observer are stated as

IF $z_{1}(t)$ is " $M_{l i}\left(z_{1}(t)\right)$ " and $\hat{z}_{2}(t)$ is " $M_{2 j}\left(\hat{z}_{2}(t)\right)$ " and $\hat{z}_{\mathbf{x}_{1}}(t)$ is " $M_{3 k}\left(\hat{z}_{x_{1}}(t)\right)$ " and $\hat{z}_{x_{2}}(t)$ is " $M_{4 l}\left(\hat{z}_{x_{2}}(t)\right)$ " and $z_{x 3}(t)$ is $M_{5 m}\left(z_{x 3}(t)\right)$ and $z_{x 4}(t)$ is ' $M_{6 n}\left(z_{x 4}(t)\right)$ "

THEN
$\hat{\dot{x}}^{R F}(t)=A_{i j k m n}^{R F} \hat{x}(t)+B_{i j k m n}^{R F} u(t)+K_{i_{-} R F}(y(t)-\hat{y}(t))+d$

$i, j, k, l, m, n=1,2$.

The aggregated fuzzy observer for the RF model turns to be

$$
\begin{aligned}
& \dot{x}^{R F}(t)=\sum_{1}^{64} h_{\psi}(\hat{z}(t))\left[A_{i j k m n}^{R F} \hat{x}(t)+B_{i j k l m n}^{R F} u(t)\right. \\
& \left.+K_{i_{-} R^{F}}(y(t)-\hat{y}(t))+d\right] \\
& \left.\hat{y}^{R F}(t)=\sum_{1}^{64} h_{\psi}(\hat{z}(t)) C \hat{x}(t)\right)
\end{aligned}
$$

\begin{tabular}{|c|c|c|c|c|}
\hline $\begin{array}{c}\text { Observer } \\
\text { gains }\end{array}$ & $x_{2}$ & $x_{2}$ & $x_{3}$ & $x_{4}$ \\
\hline $\mathrm{K}_{1 \_\mathrm{RF}}$ & -58224 & 8241.3 & 5215 & -5167.8 \\
\hline $\mathrm{K}_{2 \_\mathrm{RF}}$ & -27997 & 2342.1 & 3312 & -3319.5 \\
\hline $\mathrm{K}_{3}{ }_{\text {_RF }}^{-}$ & -56608 & 8065 & 5079.1 & -5035.7 \\
\hline $\mathrm{K}_{4-R F}$ & -26381 & 2165.8 & 3176.1 & -3187.4 \\
\hline $\mathrm{K}_{1 \_ \text {Rqel }}$ & -95553 & -5059.3 & 1637.7 & -1657.5 \\
\hline $\mathrm{K}_{2 \_ \text {Rqel }}$ & -74005 & -4888 & 1301.9 & -1338.7 \\
\hline $\mathrm{K}_{3 \text { Rqel }}$ & -75377 & -699.86 & 1257.3 & -1292.1 \\
\hline $\mathrm{K}_{4 \_ \text {Rqel }}$ & -53829 & -528.58 & 921.54 & -973.25 \\
\hline $\mathrm{K}_{1 \_ \text {Rqe2 }}$ & -44374 & 5459.5 & 3614 & -3611.4 \\
\hline $\mathrm{K}_{2 \_ \text {Rqe2 }}$ & -25288 & 1698.6 & 2603.5 & -2630.4 \\
\hline $\mathrm{K}_{3 \_ \text {Rqe2 }}$ & -42980 & 5330.1 & 3509.1 & -3509.4 \\
\hline $\mathrm{K}_{4 \_ \text {Rqe2 }}$ & -23895 & 1569.2 & 2498.6 & -2528.4 \\
\hline
\end{tabular}

where

$$
\begin{aligned}
h_{\psi}(\hat{z}(t)) & =M_{1 i}\left(\hat{z}_{1}(t)\right) M_{2 j}\left(z_{2}(t)\right) M_{3 k}\left(\hat{z}_{x_{1}}(t)\right) \\
& \times M_{4 l}\left(\hat{z}_{x_{2}}(t)\right) M_{5 m}\left(z_{x_{3}}(t)\right) M_{6 n}\left(z_{x_{4}}(t)\right)
\end{aligned}
$$

The fuzzy observers for the Rqe1 and Rqe 2 partial models were derived using the same procedure. The observer gains were calculated using the MATLAB ${ }^{\mathrm{TM}}$ Linear Matrix Inequalities (LMI's) toolbox. The observer gains for the RF, Rqe1 and Rqe2 are shown in table III.

TABLE III

FUZZY OBSERVER GAINS FOR THE RF, RQE1 AND RQE2 PARTIAL MODELS.

Also, common positive definite matrices that guarantees global asymptotic stability [10] were found for each partial model, namely

$$
\begin{gathered}
P_{R F}=\left[\begin{array}{cccc}
1.0954 \times 10^{-3} & -8.3895 \times 10^{-5} & -6.263 \times 10^{-5} & 6.0871 \mathrm{e}-005 \\
-8.3895 \times 10^{-5} & 1.1794 \times 10^{-5} & 3.8171 \times 10^{-6} & -3.7069 \times 10^{-6} \\
-6.263 \times 10^{-5} & 3.8171 \times 10^{-6} & 5.3843 \times 10^{-6} & -5.2354 \times 10^{-6} \\
6.0871 \times 10^{-5} & -3.7069 \times 10^{-6} & -5.2354 \times 10^{-6} & 5.2541 \times 10^{-6}
\end{array}\right] \\
P_{\text {Rqel }}=\left[\begin{array}{cccc}
2.3943 \times 10^{-3} & 1.0067 \times 10^{-5} & -2.7981 \times 10^{-5} & 2.6675 \times 10^{-5} \\
1.0067 \times 10^{-5} & 1.55 \times 10^{-6} & -1.3877 \times 10^{-7} & 1.3352 \times 10^{-7} \\
-2.7981 \times 10^{-5} & -1.3877 \times 10^{-7} & 3.595 \times 10^{-7} & -3.4274 \times 10^{-7} \\
2.6675 \times 10^{-5} & 1.3352 \times 10^{-7} & -3.4274 \times 10^{-7} & 3.5254 \times 10^{-7}
\end{array}\right] \\
P_{\text {Rqge2 }}=\left[\begin{array}{cccc}
2.4873 \times 10^{-3} & -1.6155 \times 10^{4} & -1.2776 \times 10^{4} & 1.2396 \times 10^{-4} \\
-1.6155 \times 10^{-4} & 2.1872 \times 10^{-5} & 6.4119 \times 10^{-6} & -6.2134 \times 10^{-6} \\
-1.2776 \times 10^{4} & 6.4119 \times 10^{-6} & 9.2698 \times 10^{-6} & -8.9975 \times 10^{-6} \\
1.2396 \times 10^{4} & -6.2134 \times 10^{-6} & -8.9975 \times 10^{-6} & 9.0991 \times 10^{-6}
\end{array}\right]
\end{gathered}
$$


However an overall common P matrix for the RF, Rqe1 and Rqe2 partial model could not be found.

\section{Simulation Results}

The application of the proposed observer scheme was simulated using MATLAB ${ }^{\mathrm{TM}}$. The fuzzy observers were tested using the fed-batch RF and the R baker's yeast partial models given in [11]. The feeding flow rate was varied in order to force the switching between both models. The initial conditions were chosen as $x_{1}(0)=0.1 \mathrm{~g} / 1, x_{2}(0)=0.02 \mathrm{~g} /$, $x_{3}(0)=0.15 \mathrm{~g} / 1, x_{4}(0)=0.0066 \mathrm{mg} / 1$ and $x_{5}(0)=3.5 \mathrm{~L}$. The behavior of the fuzzy observer for biomass estimation is shown in figure 2 . It can be noticed that the dynamics of the baker's yeast switch through the RF, Rqe1 and Rqe2 partial models and the observer converges to the real biomass values. The estimated substrate is shown in figure 3 . The observer performance is acceptable for the range of chosen values; although its performance may be degraded on initial conditions far away, from the real initial parameter value, not shown. An acceptable criteria estimation was set to \pm 5 $\%$.

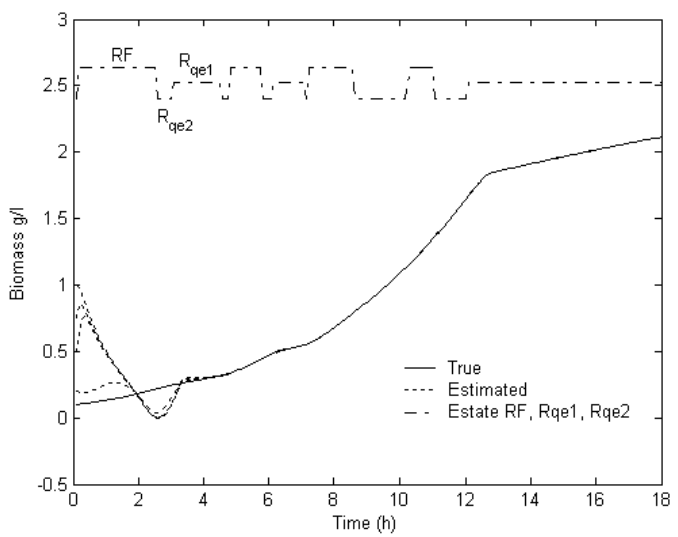

Fig. 2. Biomass estimation for the initial conditions $\hat{x}_{1}(0)=1 \mathrm{~g} / 1, \hat{x}_{1}(0)=$ $0.75 \mathrm{~g} / 1 ., \hat{x}_{1}(0)=0.5 \mathrm{~g} / 1$ and $\hat{x}_{1}(0)=0.2 \mathrm{~g} / 1$.

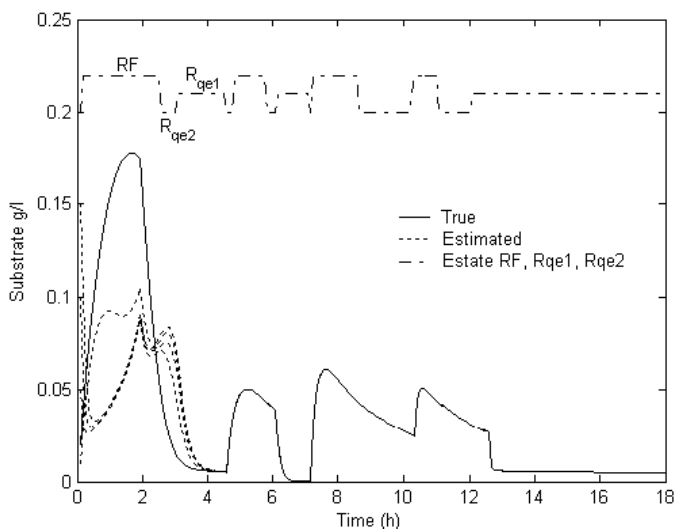

Fig.3. Substrate estimation for the initial conditions $\hat{x}_{2}(0)=0.15 \mathrm{~g} / 1, \hat{x}_{2}(0)$ $=0.1 \mathrm{~g} / 1, \hat{x}_{2}(0)=0.05$ and $\hat{x}_{2}(0)=0.01 \mathrm{~g} / 1$.

\section{CONCLUSIONS}

Based on the idea of splitting the baker's yeast model, a novel TS fuzzy model was proposed using the sector nonlinearities method, giving an exact representation of the original nonlinear plant. Moreover, an observer for each partial model was constructed. It is worth noting that the observer dynamics shows a very good tracking behavior with respect of the states of the switching partial models, without performance degradation. Therefore, the approach presented here may be considered as a valid methodology to design an observer for this class of systems. Future work will include the experimental validation of the fuzzy observer.

\section{ACKNOWLEDGMENT}

This paper has been supported by the Mexican Consejo Nacional de Ciencia y Tecnología (CONACyT), under grants 46538, 41148 and the Ph.D. Schoolarship 70662.

\section{REFERENCES}

[1] J. I. Horiuchi and M. Kishimoto, "Fuzzy-aided estimation of biological parameters based on material balances," Journal of Fermentation and Bioengineering, vol. 86, pp. 111-117, 1998.

[2] R. Oliveira, E. C. Ferreira and S. Feyo de Azevedo, "Stability, dynamics of convergence and tuning of observer-based kinetics estimators," Journal of Process Control, vol. 12, pp. 311-323, 2002.

[3] M. Arndt and B. Hitzmann, "Kalman filter based glucose control at small set points during fed-batch cultivation of Saccharomyces cerevisiae," Biotechnol. Prog., vol. 20, pp. 377-383, 2004.

[4] A. Nilsson, M. J. Taherzadeh and G. Lidén, "On-line estimation of sugar concentration for control of fed-batch fermentation of lignocellulosic hydrolyzates by Saccharomyces cerevisiae," Bioprocess Biosyst. Eng., vol. 25, pp. 183-191, 2002.

[5] S. F. Azevedo, P. Ascencio and D. Sbarbaro, "An adaptive fuzzy hybrid state observer for bioprocesses," IEEE Transactions on Fuzzy Systems, vol. 12, pp. 641-651, 2004.

[6] J. I. Horiuchi and M. Kishimoto, "Applications of fuzzy control to industrial bioprocesses in Japan," Fuzzy Sets and Systems, vol. 128, pp. $117-124,2002$.

[7] C. Karakazu, M. Türker and S. Öztürk, "Modelling on-line state estimation and fuzzy control of production scale fed-batch baker's yeast fermenttion," Control Engineering Practice, vol. 14, pp. 959974, 2006.

[8] M. Chidambaram and B. Satishkumar, "Control of unstable bioreactor using fuzzy tuned PI controller," Bioprocess Engineering., vol. 20, pp. 127-132, 1999.

[9] T. Takagi and M. Sugeno, "Fuzzy identification of systems and its applications to modeling and control," IEEE Trans. Sys, Man Cyber, vol. 15 , pp. 116-132, 1985 .

[10] K. Tanaka and H. Wang, Fuzzy Control Systems Design and Analysis, A Linear Matrix Inequality Approach, John Wiley \& Sons, United States of America, 2001.

[11] E. C. Ferreira, Identificação e controlo adaptivo de processos biotecnológicos), Ph.D. Dissertation, Universidade do Porto, Portugal 1995.

[12] P. Pormeleau, Modelisation et controle d'un procéde fed-batch de culture des levures a pain (saccharomyces cerevisiae), Ph.D. Dissertation, Ecole Polytechnique de Montréal, Canada, 1990.

[13] B. Sonnleitner, and O. Käppeli, "Growth of saccharomyces cerevisiae is controlled by its limited respiratory capacity: formulation and verification of a hypothesis," IEEE Trans. Biotechnology and Bioengineering, vol. 28, pp. 927-937, 1986. 